\title{
Synthesis and Characterization of Antibacterial Viscose Nonwoven Fabric by the Cooperative Action of Se Nanoparticles and Amino Hyperbranched Polymer
}

\author{
Yao Xiao \\ Nantong University \\ Zhenzheng Gan \\ Nantong University \\ Erying Dong \\ Nantong University \\ Jiawei Yan \\ Shinshu Daigaku \\ Wanwan Liu \\ Nantong University \\ Guangyu Zhang ( $\square$ zgyu85@ntu.edu.cn ) \\ Nantong university https://orcid.org/0000-0002-1364-3350
}

\section{Research Article}

Keywords: Viscose nonwoven fabric, Selenium nanoparticles, Antibacterial

Posted Date: December 17th, 2021

DOI: https://doi.org/10.21203/rs.3.rs-1153047/v1

License: (c) (i) This work is licensed under a Creative Commons Attribution 4.0 International License.

Read Full License 


\section{Abstract}

This research provides a new method for preparing nanoparticle-coated cellulose fabrics, which has broad application prospects in the functional fiber industry. In this work amino-terminated hyperbranched poly (HBP)-capped Selenium nanoparticles (Se NPs) were synthesized for coating viscose nonwoven fabric (VNF) via impregnation method to produce a controllable and uniform Se NPs coating on the viscose fiber surface. The prepared Se NPs and the treated VNF were characterized by the transmission electron microscope (TEM), x-ray diraction (XRD), x-ray photoelectron spectroscopy (XPS), field emission scanning electron microcopy (FE-SEM), and antibacterial measurement. The results indicate that the Se NPs were spherical shaped with an average size of $10 \mathrm{~nm}$. FESEM, XRD, and XPS characterizations demonstrated that Se NPs can adsorbed and distributed uniformly on the fiber surface. Se NPs-coated VNF showed above $99.9 \%$ bacterial reduction of Staphylococcus aureus and Escherichia coli while the Se element content on VNF was about $292 \mathrm{mg} / \mathrm{kg}$.

\section{Introduction}

As a regenerated cellulose fiber, viscose is made from a cellulose solution, such as cotton linters, bamboo, or wood by wet-spinning method (Abou-Okeil et al. 2020; Kebede, et al. 2017). In the textile industry, unlike the woven and knitted viscose fabric, viscose nonwoven fabric (VNF) was produced with viscose fibers by web-forming and fixing technology ( Liu et al. 2021; Liu et al. 2020; Zhang et al. 2021). Given the excellent hygroscopicity, softness, degradability, and good air permeability, VNF has an important role in medical care applications. However, because of its large surface area and ability to retain moisture in the fabric grid, VNF also provides an excellent environment for microbial reproduction, limiting its application. With the growing concern about public health, antimicrobial cellulose products have attracted much attention, especially for health care materials used in medical occupations (Montaser et al. 2021; Pathania et al. 2015; Qu et al. 2019; Sun et al. 2020; Yang et al. 2021). Therefore, many functional agents, such as metallic nanoparticles (NPs) and plant extracts, have been employed to improve antimicrobial activity of cellulose materials (Pathania et al. 2015; Rehan et al. 2018). For instance, Varaprasad et al. (2016) synthesized zinc oxide nanoparticles by precipitation method and were coated on the surface of cellulose fiber. The modified cellulose fiber showed excellent antimicrobial activity. Zheng et al. (2014) synthesized acrylate copolymer and used it for the preparation of Ag nanoparticles on the surface of viscose fiber. The modified viscose fibers were endowed with remarkable antimicrobial activity even after several washing cycles. Sun et al. (2020) prepared wormwood extract/viscose spun laced nonwoven fabric using waterborne polyurethane to improve the antibacterial activity of the cellulose materials.

Further to the above-mentioned antimicrobial materials, selenium (Se) NPs are recognized as safe materials and have a good potential to inhibit the growth of bacteria, viruses, fungi, and other microorganisms ( Liu et al. 2019; Srivastava et al. 2015; Stevanović et al. 2015; Ye et al. 2020; Zhang et al. 2019). Se is an essential trace element for human body, which has the characteristics of enhancing body immunity, high biological activity, and biocompatibility, and can highly acceptable by human 
cells (Chen et al. 2015; Sadalage et al. 2020; Tang et al. 2020). In recent years, studies have shown that Se NPs show good antibacterial activity against the Staphylococcus aureus (S. aures) and Escherichia coli (E. coil) (Biswas et al. 2018; Phong et al. 2011; Wadhwani et al. 2016). Phong Atran et al (2013) used Se NPs in the coating of polymer medical devices to protect medical devices from infection. Biswas et al (2018) investigated the loading of Se NPs into porous chitosan/PVA (CS) scaffolds through in situ deposition method to create distinct wound dressing materials (CS-Se). Se-loaded scaffolds showed abilities to damage bacterial cell membrane and nontoxicity to fibroblast. Chung et al (2016) prepared electrospun silk scaffolds doped with Se NPs. Results showed significantly improved bacterial inhibition and mild improvement in human dermal fibroblast metabolic activity.

However, very few research concerns on the synthesis of Se NPs on the fibers surface were noticed. For example, Se NPs were also prone to aggregation into large clusters in aqueous solution, leading to a lower bioactivity and bioavailability. To solve this problem, the researchers found that polysaccharides and hyperbranched polymer can modify the interface of Se NPs, control the growth, and stabilize the solution of Se NPs. For another, Se NPs lack of binding force with the fiber, during the finishing process, some binders, such as polyurethane resin polyacrylic acid and citric acid were required to fix the Se NPS on the fibers (Tang et al. 2020; Wadhwani et al. 2016). Therefore, the softness, air permeability, and hygroscopicity of the treated fiber may affected by these chemicals.

In our previous study(Zhang et al. 2013), amino hyperbranched polymer (HBP) was successfully designed and applied to the control synthesis of metallic NPs. The prepared metallic NPs can completely be adsorbed on cellulose fibers depending on the strong molecular interactions between the amino HBP and the hydroxyl on the cellulose fiber surfaces. In this study, Se NPs coating was formed by the circular introduction of HBP to the VNF surface through a simple cyclic impregnation method. The physicalchemical properties of the VNF were evaluated by Fourier transform infrared spectroscopy (FTIR), Field scanning electron microscopy (FE-SEM), x-ray photoelectron spectroscopy (XPS), and x-ray diffraction (XRD). The antibacterial activity of the Se NPs-coated VNF against Escherichia coli (E. coli) and Staphylococcus aureus (S. aureus) were measured quantitatively.

\section{Experimental}

\subsection{Materials}

VNF (Spunlace reinforcement) with a basic of $60 \mathrm{~g} / \mathrm{m}^{2}$ was obtained from Jinsanfa Co., Ltd (Zhejiang, china). Sodium selenite $\left(\mathrm{Na}_{2} \mathrm{SeO}_{3}\right)$ and ascorbic acid $(\mathrm{Vc})$ were purchased from Sinopharm Chemical Technology Co., Ltd. Amino Hyperbranched polymers (HBP) are prepared as described in our previous paper. S. aureus (ATCC 6538) and E. coli (ATCC 8099) were obtained from the Shanghai Luwei Technology Co., Ltd. (China).

2.2 Synthesis of Se NPs 
Scorbic acid solution ( $\mathrm{Vc}$ ) with concentration of $1 \mathrm{mM}$ was dropwise in the $\mathrm{Na}_{2} \mathrm{SeO}_{3}$ and $\mathrm{HBP}$ solutions, where the mixture was stirred with a magnetic stirrer for three hours. The concentration of $\mathrm{Na}_{2} \mathrm{SeO}_{3}$ in the reaction system was $0.05 \mathrm{mM}$ and $0.1 \mathrm{mM}$, the molar ratio of $\mathrm{Na}_{2} \mathrm{SeO}_{3}$ and $\mathrm{Vc}$ was maintained at 1:3, and the concentration of HBP is $2 \mathrm{~g} / \mathrm{L}$. F; hence prepared by centrifugation at $8000 \mathrm{rpm}$ for ten minutes to produce precipitation.

\subsection{Preparation of Se NPs-Coated VNF}

To prepare Se NPs aqueous solution, $0.05 \mathrm{mM}, 0.1 \mathrm{mM}$, and $0.15 \mathrm{mM} \mathrm{Na}_{2} \mathrm{SeO}_{3}$ were added into the $2 \mathrm{~g} / \mathrm{L}$ HBP solution. Vc with concentration of $1 \mathrm{mM}$ was added dropwise in the mixing solution with the molar ratio of $\mathrm{Na}_{2} \mathrm{SeO}_{3}$ and $\mathrm{Vc}$ at 1:3. Afterward, $1 \mathrm{~g} \mathrm{VNF}$ was dropped into the mixing solution with bath ratio 1:50 in a constant temperature water bath at $30{ }^{\circ} \mathrm{C}, 50{ }^{\circ} \mathrm{C}$, and $90{ }^{\circ} \mathrm{C}$ for two hours. The Se NPs-coated VNF was thermally pretreated at $80^{\circ} \mathrm{C}$ for five minutes and then at $150{ }^{\circ} \mathrm{C}$ for three minutes.

\subsection{Characterization of Se NPs and Se NPs-coated VNF}

The morphology and lattice characteristics of Se NPs were characterized by transmission electron microscopy (TEM) (2010, JEOL, Tokyo, Japan). The crystalline phases of Se NPs and Se NPs-coated VNF were analyzed by XRD (Philips, Amsterdam, The Netherlands) via a CuKa x-ray source at a voltage and current of $40 \mathrm{kV}$ and $30 \mathrm{~mA}$, respectively. FTIR spectroscopy (NICOLET 6700), XRD (Philips, Amsterdam, Netherlands), and XPS were used to analyze the viscose nonwoven fabric before and after modification. The morphology and elements of viscose nonwovens were studied by FE-SEM (Scios DualBeam, Czechia) and energy dispersive spectrometer (EDS) (Carl Zeiss, EV015, Oberkochen. The antibacterial test uses E.coli (AATCC 1555) and S. aureus (AATCC 547) as microbial models. According to GB/T20944.32008 , the antibacterial activity of VNF loaded with Se NPs against E.coli and S. aureus was determined by the shaking method.

\section{Results And Discussion}

\section{Formation mechanism of Se Nanoparticles}

This experiment uses $\mathrm{V}_{\mathrm{C}}$ as the reducing agent to reduce $\mathrm{SeO}_{3}{ }^{2-}$ to $\mathrm{Se}^{0}$. The reaction can be clearly observed from the color change. The color of the solution gradually changed from colorless to light yellow to orange and then to red. The appearance of the red color characteristic indicates that a reaction to form amorphous or monoclinic selenium particles has taken place. Sch. 1 shows that the synthesis of Se NPs may begin with an electrostatic combination of precursor $\left(\mathrm{SeO}_{3}{ }^{2-}\right)$ and amino groups of the HBP. When adding the $\mathrm{V}_{\mathrm{c}}$ in the mixing solution, $\mathrm{SeO}_{3}{ }^{2-}$ can be reduced to $\mathrm{Se}^{0}$. ( Zhang et al. 2004; Zhang et al. 2010)The HBP was used as a stabilizer to solve the problem of easy agglomeration of Se NPs mainly because HBP contains many cavities inside, which is a typical molecular cage structure, and the reduced selenium atoms enter the molecular cage. As more selenium atoms are reduced in the reaction and when larger nanocrystals are formed, the growth of Se NPs particles are restricted by the molecular cage, 
thereby forming stable growth and nonagglomerated Se NPs. Given the protection of HBP, the prepared Se NPs has good stability and not aggregated for two weeks.

To further explore the morphologies, TEM was used to depict the shape and size of the Se NPs. The TEM images of the prepared Se NPs are shown in Fig. 1. The results showed that Se NPs were well-dispersed spherical particles in the solution (Fig. 1 (a)), and the diameter of Se NPs was about $20 \mathrm{~nm}$. The crystalline phase of the prepared Se NPs was characterized by the powder XRD method, as shown in Fig. 1 (b). XRD analysis of the prepared Se NPs showed a clear and sharp Bragg reflection. The Bragg reflection peaks at $2 \theta=23.5,29.7,41.2,43.6,45.3,51.5,55.7,61.6,65.1$, and 68.2 were attributed to the (100), (101), (110), (102), (111), (201), (003), (202), (210), and (211), respectively. All peaks correspond to the planes of pure hexagonal phase of Se crystal and well agreement with JCPDS data (06-0362)(Kaur et al. 2009; Stevanović et al. 2015; Ye et al. 2020).

\section{Formation mechanism of Se Nps-coated VNF}

VNF was immersed in the colloidal solution of HBP/Se NPs to obtain the Se NPs-coated VNF, and the mechanism is shown in Sch. 2. When immersing in the HBP/Se NPs mixing solution, HBP/Se NPs can be coated onto the cellulose fiber in a very short time because it can be attributed to large number of amino groups on the surface of Se NPs combined with viscose fibers through intermolecular hydrogen bonds between amino of HBP and carboxyl groups on the cellulose fibers. The electrostatic bond interaction between the negatively charged hydroxyl groups on the cellulose fibers and the positively charged amino groups can also enhance the stability of the Se NPs on the surface of cellulose fiber. (Xu et al. 2016; Xu et al. 2017) The intermolecular interaction is much stronger than Van der Waals force, thus the prepared Se NPs coating shows higher firmness than traditional methods.

The electrostatic bond interaction between the VNF and Se NPs can be verified by testing the zeta potential values. To confirm the zeta potential values of HBP/Se NPs and VNF, the sample was tested under different $\mathrm{pH}$ conditions, as shown in Fig. 2. The isoelectric points of VNF and HBP/Se NPs were about 4.0 and 9.4, respectively. Since the surface of cellulose contains many hydroxyl groups, when VNF was immersed in the solution at $\mathrm{pH}$ values above 4.0, the electrolysis of hydroxyl groups makes the surface of the VNF negatively charged. The positive charge of HBP/Se NPs may have resulted from the presence of abundant imino groups and terminal primary amino groups in the HBP, which gained $\mathrm{H}^{+}$ions in the liquid phase, and then turned into cationic groups at pHs lower than 9.4. As the HBP/Se solution obtained a pH value of 9.1, Se NPs showed positive charge in HBP aqueous solution, while the VNF exhibited negative charge. Therefore, besides the hydrogen bonding, Se NPs can be actively adsorbed on the surface of VNF by electrostatic adsorption.( Zhang et al. 2015)

As Se NPs have large surface energy, dispersing and combining it with VNF is difficult. To provide the Se NPs-coated VNF and impart the VNF with antibacterial properties, the VNF was treated with the aqueous solution of HBP/Se NPs by the impregnation method at $60^{\circ} \mathrm{C}$ and the concertation of Se element was $0.05 \mathrm{mM}, 0.1 \mathrm{mM}$, and $0.15 \mathrm{mM}$. As shown in Fig. 3 (a), VNF with clean and smooth surface has an 
obvious change between the VNF and Se NPs treated VNF, where the Se NPs treated sample of Fig. 3 (bd) became rough, and parts of Se NPs can be found on the surface of treated VNF. When the concentration of Se NPs is $0.05 \mathrm{mM}$, the number of Se NPs on the fiber surface is less. More Se NPs can be found on the surface and the nanoparticles were well-dispersed with increased concentration of Se NPs at $0.1 \mathrm{mM}$ and $0.15 \mathrm{mM}$. The contents of Se NPs were further characterized by ICP method, as shown in Fig.S1. The amount of attached Se NPs on the surface of increased VNF with increasing Se concentration results in the adsorbing capacity of about $290 \mathrm{mg} / \mathrm{g}$. However, when the concentration of Se is from $0.1 \mathrm{mM}$ to $0.15 \mathrm{mM}$, the contents of Se on VNF showed little difference.

In the preparation process of Se NPs-coated VNF, the reaction temperature has also influenced the loading of Se NPs. Fig. 4 showed the morphology of the treated VNF by $0.1 \mathrm{mM}$ Se NPs at different treating temperature. An obvious change between the Se NPs treated VNF at different temperature was observed, as shown in Fig. 4 (a). Very few nanoparticles can be found at $30{ }^{\circ} \mathrm{C}$ treatment. With increasing the reaction temperature, more Se NPs can be found. Fig. 4 (b) shows that when VNF is treated by $50{ }^{\circ} \mathrm{C}$ $\mathrm{HBP} / \mathrm{Se}$ aqueous solution, the Se NPs on the surface of VNF were mainly spherical structures. The morphology of the Se NPs on the VNF change at the reaction temperatures of $70{ }^{\circ} \mathrm{C}$ and $90{ }^{\circ} \mathrm{C}$ (Fig. 4 (c), (d)) because of the adsorption between the amino group and $\mathrm{SeO}_{3}{ }^{2-}$, which hinders the mutual contact and collision of the reactant molecules, thereby slowing down the reaction speed. When the temperature is low, the nucleation rate in the solution is greater than the growth rate, which is conducive to obtaining small selenium crystal grains. The viscosity of the solution decreased above $90^{\circ} \mathrm{C}$, the ability of HBP to control the growth of Se NPS crystals was weakened, the temperature increase accelerated the growth rate of Se crystals, and the morphology of Se NPs is combination of spherical and rod-shaped. VNF and VNF treated by $0.1 \mathrm{mM}$ Se NPs at $60^{\circ} \mathrm{C}$ were further characterized by the FTIR method, as shown in Fig. S2. The peak of Se NPs-coated VNF at $1650.79 \mathrm{~cm}^{-1}$ belongs to the acetamide groups, and the characteristic absorption peaks of hydroxyl group peak of Se NPs-coated VNF at $3340 \mathrm{~cm}^{-1}$ are larger than the VNF attribute to the amino group of HBP. The change of absorption peaks may indicate an interaction between hydroxyl groups of VNF and surface of Se NPs.

The elemental distributions on VNF were further investigated using the EDS spectra and elemental maps of the treated VNF, as shown in Fig. 5a and Fig.S3. The treated VNF contains C, O, and Se. The observed Se elements can confirm the syneresis of Se on the surface of VNF. The surface scanning images shown in Fig. $5(b-d)$ show that $O$ and $C$ elements of the cellulose fiber as well as the even distribution of Se on the VNF surface are found, which confirmed the possibility of the existence of Se element on the VNF. The surface scanning images are in good agreement with the SEM measurement.

To confirm the Se NPs which were combined with VNF, the XRD patterns of the control and treated VNF were tested, as shown in Fig. 6. The diraction peaks at $2 \theta$ values of 12.2, 20.5 and 22.1 as shown in Fig. 5 $(a, b)$, which are assigned to the (1-01), (110) and (020) planes of cellulose II from viscose filaments( French et al. 2014; Yao et al. 2020; Zhu et al. 2000). In contrast, the treated VNF (Fig. 5b) has reflection peaks at $2 \theta$ values of $23.5,29.7,41.2,43.6$, and 45.3 , which are indexed to the (100), (101), (110), (102), 
(111), and (201) planes of the hexagonal phase of Se crystal, respectively (Fig. 5c) and well agreement with JCPDS data (06-0362). (Nakhowong et al. 2021; Ye et al. 2020)

XPS analysis was performed to identify the chemical state of Se element in VNF. Fig. 7a shows that VNF fibers displayed peaks of 01s and C1s. New Se3d peaks were observed after treated with Se NPs (Fig. 7a), indicating the coating of Se element on the VNF fiber. Fig. 7 shows the Se3d spectra. The binding energies of two peaks were at 55.9 and $55.1 \mathrm{eV}$, corresponding to the Se3d and presents an unambiguous proof that the nanoparticles on the VNF are indeed Se0 (Se NPs).( Liu et al. 2019; Wu et al. 2021)

\section{Antibacterial properties and mechanical properties of Se NPs-coated VNF}

$S$. aureus and E. coli representatives were selected to determine the antimicrobial activity of the Se and Se NPs-coated VNF. The antibacterial properties of Se NPs were determined by disk diffusion method. The results are illustrated in Fig. S4 and Fig. S5. Obvious inhibition zones around the oxford cups loaded with $0.05 \mathrm{mM}$ and $0.01 \mathrm{mM}$ Se NPs which indicated that Se NPs have effective antibacterial activities against S. aureus and E. coli are observed Fig. S6 and Tab. 1 show the bacterial count of $S$. aureus and E. coli colonies of the VNF. Given the abundant amino groups of HBP, HBP treated VNF shows the antibacterial activity for the $S$. aureus and $E$. coil, and the ratios of the antibacterial activity are less than $40 \%$. After coating with Se NPs, the VNF presented excellent antibacterial activity for both the $S$. aureus and $E$. coil representative samples, and the ratios of the antibacterial activity reached more the $99.9 \%$. Antimicrobial mechanism of Se NPs-coated VNF was shown in Sch 3, the Se NPs exchange bacterial cell appearance and membrane structure, change in permeability of the bacterial layer, and generated oxidative stress for the response genes in the bacteria due to the production of $\mathrm{H}_{2} \mathrm{O}_{2}$. (Chaudhary et al. 2018; Raahati et al. 2020; Xu et al. 2021) HBP are positively charged, S. aureus and E. coli are usually negatively charged, and the electrostatic interaction between the bacteria and HBP can inhibit the growth of bacteria. HBP combined with Se NPs has synergy antibacterial activities. When contacting with HBP/Se NPs, the growth of bacteria is limited and the Se NPs-coated VNF shows excellent antibacterial properties.

Table 1

Antibacterial activity of Se and Se NPs coated-VNF

\begin{tabular}{|c|c|c|c|c|c|}
\hline \multirow[t]{2}{*}{ Sample } & \multirow{2}{*}{$\begin{array}{l}\text { Se content } \\
(\mathrm{mg} / \mathrm{kg})\end{array}$} & \multicolumn{2}{|l|}{ S. aureus } & \multicolumn{2}{|l|}{ E. coli } \\
\hline & & $\begin{array}{l}\text { Surviving cells } \\
\text { (CFU/mL) }\end{array}$ & $\begin{array}{l}\text { Reduction } \\
\text { (\%) }\end{array}$ & $\begin{array}{l}\text { Surviving cells } \\
\text { (CFU/mL) }\end{array}$ & $\begin{array}{l}\text { Reduction } \\
\text { (\%) }\end{array}$ \\
\hline VNF & - & $1.38 \times 10^{6}$ & - & $3.1 \times 10^{5}$ & - \\
\hline HBP/VNF & - & $9.6 \times 10^{5}$ & 30.43 & $2.01 \times 10^{5}$ & 35.16 \\
\hline Se/VNF & 290 & $\bigotimes 10$ & 99.99 & $\$ 10$ & 99.99 \\
\hline
\end{tabular}

Viscose is produced by dissolving cellulose, then converting the solution back to insoluble fibrous cellulose. Compared with cotton fiber, the crystallinity and orientation of viscose fiber are low. In wet condition, the strength of cellulose fiber drops more for about $50 \%$ of the dry strength, and the wet 
elongation increases by about 50\%.(Chandrasekaran et al. 2018; Oner et al. 2019; Rajan et al. 2018) Using the padding method, the viscose fiber is easy to deform under small load, and the impregnation method is a good choice for the treatment of viscose. The mechanical properties of viscose and Se NPscoated VNF was measured, with VNF treated by HBP without Se NPs as reference sample. The VNF is made with viscose fiber after carding and spunlace reinforcement, and the viscose fiber in the VNF exhibit vertical arrangement. As shown in Fig. 8, the longitudinal strengths of VNF with $730 \mathrm{~N}$ is much higher than the cross-breaking strengths about $260 \mathrm{~N}$. When treated by Se NPs and HBP, the longitudinal and cross-breaking strengths of VNF have declined to $700 \mathrm{~N}$ and $233 \mathrm{~N}$, respectively. The treatment process received a minimal effect on the longitudinal and cross-mechanical proper ties of Viscose NWFs.

\section{Conclusion}

Se NPs were successfully prepared in $\mathrm{Na}_{2} \mathrm{SO}_{3}, \mathrm{Vc}$ and $\mathrm{HBP}$ mixing solution. The average diameter of the Se NPs was about $20 \mathrm{~nm}$. The prepared Se NPs showed good stability and dispersion and not obviously aggregated in two weeks. Vc as a reducing agent and HBP as a stabilizing agent during the synthetic process. The VNF was treated by Se NPs through an impregnation method with different concertation and temperature. All results of FE-SEM, ICP-AES, FTIR, and XPS determinations confirmed that Se NPS have been fixed and well dispersed on VNF surface through chemical and electrostatic interactions between the hydroxyl and amino groups. The Se NPs-coated VNF fabrics showed more than $99.9 \%$ antibacterial properties against both $S$. aureus and $E$. coli at the content of $290 \mathrm{mg} / \mathrm{Kg}$. The mechanical property of VNF and Se NPs treated VNF have not changed very much.

\section{Declarations}

\section{Declaration of Competing Interest}

The authors declare that they have no known competing financial interests or personal relationships that could have appeared to influence the work reported in this paper.

\section{Acknowledgments:}

This work was supported by the National Science Foundation (Grant No. 51503105), Education Department of Jiangsu Province "Qinglan project" and Research Innovation Program for College Graduates of Jiangsu Province (Grant No. KYCX20_2834)

\section{References}

1. Abou-Okeil A, Sheta AM, Amr A, Ali M (2012) Wound dressing based on nonwoven viscose fabrics. Carbohydr Polym 90(1):658-666 https://doi.org/10.1016/j.carbpol.2012.05.093

2. Bhuiyan MAR, Wang L, Shanks RA, Ara ZA, Saha T (2020) Electrospun polyacrylonitrile-silica aerogel coating on viscose nonwoven fabric for versatile protection and thermal comfort. Cellulose 
27(17):10501-10517. https://doi.org/10.1007/s10570-020-03489-9

3. Biswas DP, O'Brien-Simpson NM, Reynolds EC, O'Connor AJ, Tran PA (2018) Comparative study of novel in situ decorated porous chitosan-selenium scaffolds and porous chitosan-silver scaffolds towards antimicrobial wound dressing application. J Colloid Interface Sci 515:78-91. https://doi.org/10.1016/j.jcis.2018.01.007

4. Chandrasekaran V, Senthilkuma P, Sakthivel JC (2018) Study on moisture management properties of Micro-Pore Ring-Spun Viscose Yarn-plated knitted fabrics. J Text Inst 109(11):1458-1464. https://doi.org/10.1080/00405000.2018.1423884

5. Chaudhary S, Chauhan P, Kumar R, Bhasin KK (2018) Toxicological responses of surfactant functionalized selenium nanoparticles: A quantitative multi-assay approach. Sci Total Environ 643:1265-1277. https://doi.org/10.1016/j.scitotenv.2018.06.296

6. Chen W, Li Y, Yang S, Yue L, Jiang Q, Xia W (2015) Synthesis and antioxidant properties of chitosan and carboxymethyl chitosan-stabilized selenium nanoparticles. Carbohydr Polym 132:574-581. https://doi.org/10.1016/j.carbpol.2015.06.064

7. Chung S, Ercan B, Roy AK, Webster TJ (2016) Addition of selenium nanoparticles to electrospun silk scaffold improves the mammalian cell activity while reducing bacterial growth. Front Physiol 7:297. https://doi.org/10.3389/fphys.2016.00297

8. French AD (2014) Idealized powder diffraction patterns for cellulose polymorphs. Cellulose 21:885896. https://doi.org/10.1007/s10570-013-0030-4

9. Kaur G, Iqbal M, Bakshi MS (2009) Biomineralization of Fine Selenium Crystalline Rods and Amorphous Spheres. J Phys Chem C 113(31):13670-13676. https://doi.org/10.1021/jp903685g

10. Kebede MA, Imae T, Wu CM, Cheng KB (2017) Cellulose fibers functionalized by metal nanoparticles stabilized in dendrimer for formaldehyde decomposition and antimicrobial activity. Chem Eng $\mathrm{J}$ 311:340-347 https://doi.org/10.1016/j.cej.2016.11.107

11. Liu F, Hua S, Zhou L, Hu B (2021) Development and characterization of chitosan functionalized dialdehyde viscose fiber for adsorption of $\mathrm{Au}(\mathrm{III})$ and $\mathrm{Pd}(\mathrm{II})$. Int J Biol Macromol 173:457466 https://doi.org/10.1016/j.jibiomac.2021.01.145

12. Liu LJ, Xiao ZP, Niu S, He YD, Wang G, Pei X (2019) Preparation, characteristics and feeble inducedapoptosis performance of non-dialysis requiring selenium nanoparticles@chitosan. Mater Design 182:10. https://doi.org/10.1016/j.matdes.2019.108024

13. Liu Y, Tao Y, Wang B, Li P, Xu YJ, Jiang ZM (2020) Fully bio-based fire-safety viscose/alginate blended nonwoven fabrics: thermal degradation behavior, flammability, and smoke suppression. Cellulose 27(10):6037-6053. https://doi.org/10.1007/s10570-020-03162-1

14. Montaser AS, Jlassi K, Ramadan MA, Selem AA, Attia MF (2021) Alginate, gelatin, and carboxymethyl cellulose coated nonwoven fabrics containing antimicrobial AgNPs for skin wound healing in rats. Int J Biol Macromol 173:203-210 https://doi.org/10.1016/j.jibiomac.2021.01.123

15. Nakhowong R, Kaewchin W, Chueachot R (2021) Reduction in electrical resistivity and enhancing the thermoelectric power factor of $\beta$-FeSi2 bulk materials by Se doping. Mater Lett 302:130408. 
https://doi.org/10.1016/j.matlet.2021.130408

16. Oner E (2019) Mechanical and Thermal Properties of Knitted Fabrics Produced from Various Fiber Types. Fibers Polym 20(11):2416-2425. https://doi.org/10.1007/s12221-019-9119-1

17. Pathania D, Kumari M, Gupta VK (2015) Fabrication of ZnS-cellulose nanocomposite for drug delivery, antibacterial and photocatalytic activity. Mater Design 87:1056-1064. https://doi.org/10.1016/j.matdes.2015.08.103

18. Qu J, He N, Patil SV, Wang Y, Banerjee D, Gao W (2019) Screen Printing of Graphene Oxide Patterns onto Viscose Nonwovens with Tunable Penetration Depth and Electrical Conductivity. ACS Appl Mater Interfaces 11(16):14944-14951 https://doi.org/10.1021/acsami.9b00715

19. Raahati Z, Bakhshi B, Najar-peerayeh S (2020) Selenium Nanoparticles Induce Potent Protective Immune Responses against Vibrio cholerae WC Vaccine in a Mouse Model. Journal of Immunology Research, 2020. https://doi.org/10.1155/2020/8874288

20. Rajan R, Rainosalo E, Ramamoorthy SK, Thomas SP, Zavasnik J, Vuorinen J, Skrifvars M (2018) Mechanical, thermal, and burning properties of viscose fabric composites: Influence of epoxy resin modification. J Appl Polym Sci 135(36):12 https://doi.org/10.1002/app.46673

21. Rehan M, Khattab TA, Barohum A, GätjenL, Wilken R (2018) Development of $A g / A g X(X=C l, I)$ nanoparticles toward antimicrobial, UV-protected and self-cleanable viscose fibers. Carbohydr Polym 197:227-236. https://doi.org/10.1016/j.carbpol.2018.06.010

22. Sadalage PS, Nimbalkar MS, Sharma KKK, Patil PS, Pawar KD (2020) Sustainable approach to almond skin mediated synthesis of tunable selenium microstructures for coating cotton fabric to impart specific antibacterial activity. J Colloid Interface Sci 569:346-357. https://doi.org/10.1016/j.jcis.2020.02.094

23. Srivastava N, Mukhopadhyay M (2015) Green synthesis and structural characterization of selenium nanoparticles and assessment of their antimicrobial property. Bioprocess Biosyst Eng 38(9):17231730. https://doi.org/10.1007/s00449-015-1413-8

24. Stevanović M, Filipović N, Djurdjević J, Lukić M, Milenković M, Boccaccini A (2015) 45S5Bioglass®based scaffolds coated with selenium nanoparticles or with poly (lactide-co-glycolide)/selenium particles: Processing, evaluation and antibacterial activity. Colloids Surf, B 132:208215 https://doi.org/10.1016/j.colsurfb.2015.05.024

25. Sun L, Feng Y, Qian X (2020) Preparation and properties of wormwood extract/viscose spunlaced nonwovens. The Journal of The Textile Institute 112:707709 https://doi.org/10.1080/00405000.2020.1776531

26. Tang HY, Huang Q, Wang YL, Yang XQ, Su DX, He S (2020) Development, structure characterization and stability of food grade selenium nanoparticles stabilized by tilapia polypeptides. J Food Eng 275:109878. https://doi.org/10.1016/j.jfoodeng.2019.109878

27. Tran PA, Webster TJ (2011) Selenium nanoparticles inhibit Staphylococcus aureus growth. Int J Nanomed 6:1553. https://doi.org/10.2147/IJN.S21729 
28. Tran PA, Webster TJ (2013) Antimicrobial selenium nanoparticle coatings on polymeric medical devices. Nanotechnology 24(15):155101 https://doi.org/10.1088/0957-4484/24/15/155101

29. Varaprasad K, Raghavendra GM, Jayaramudu T, Seo J (2016) Nano zinc oxide-sodium alginate antibacterial cellulose fibres. Carbohydr Polym 135:349-355.

https://doi.org/10.1016/j.carbpol.2015.08.078

30. Wadhwani SA, Shedbalkar UU, Singh R, Chopade BA (2016) Biogenic selenium nanoparticles: current status and future prospects. Appl Microbiol Biotechnol 100(6):2555-2566

31. Wu H, Chen J, Xu L, Guo X, Fang P, Du K, Sheng G (2021) Decorating nanoscale FeS onto metalorganic framework for the decontamination performance and mechanism of $\mathrm{Cr}(\mathrm{VI})$ and $\mathrm{Se}(\mathrm{IV})$. Colloids Surf, A 625:126887 https://doi.org/10.1016/j.colsurfa.2021.126887

32. Xu S, Chen S, Zhang F, Jiao C, Song J, Chen Y, Morikawa H (2016) Preparation and controlled coating of hydroxyl-modified silver nanoparticles on silk fibers through intermolecular interaction-induced self-assembly. Mater Design 95:107-118. https://doi.org/10.1016/j.matdes.2016.01.104

33. Xu SJ, Zhang F, Yao LR, Zhu CH, Morikawa H, Chen YY (2017) Eco-friendly fabrication of antibacterial cotton fibers by the cooperative self-assembly of hyperbranched poly(amidoamine)and hyperbranched poly(amine-ester)-functionalized silver nanoparticles. Cellulose 24(3):14931509. https://doi.org/10.1007/s10570-016-1178-5

34. Xu XT, Zhang HM, Gao TY, Teng JH, Lu MY (2021) Antibacterial thin film nanocomposite forward osmosis membranes produced by in-situ reduction of selenium nanoparticles. Process Saf Environ Prot 153:403-412 https://doi.org/10.1016/j.psep.2021.07.037

35. Yang RH, Pan B, Wang LJ, Li JW (2021) Blending effects and performance of ring-, rotor-, and air-jetspun color-blended viscose yarns. Cellulose 28:1769-1780 https://doi.org/10.1007/s10570-02003601-z

36. Yao W, Weng Y, Catchmark JM (2020) Improved cellulose X-ray diffraction analysis using Fourier series modeling. Cellulose 27(10):5563-5579. https://doi.org/10.1007/s10570-020-03177-8

37. Ye X, Chen Z, Zhang Y, Mu J, Chen L, Li B, Lin X (2020) Construction, characterization, and bioactive evaluation of nano-selenium stabilized by green tea nano-aggregates. LWT 129:109475 https://doi.org/10.1016/j.lwt.2020.109475

38. Zhang C, Liu Y (2021) Selective adsorption of zwitterionic viscose fiber treated with sodium chloroacetate and hyperbranched polyethylenimine. Iran Polym J 30(1):57-65. https://doi.org/10.1007/s13726-020-00872-y

39. Zhang G, Liu Y, Morikawa H, Chen Y (2013) Application of ZnO nanoparticles to enhance the antimicrobial activity and ultraviolet protective property of bamboo pulp fabric. Cellulose 20(4):1877-1884. https://doi.org/10.1007/s10570-013-9979-2

40. Zhang SY, Zhang J, Wang HY, Chen HY (2004) Synthesis of selenium nanoparticles in the presence of polysaccharides. Mater Lett 58(21):2590-2594 https://doi.org/10.1016/j.matlet.2007.01.095

41. Zhang W, Zhang D, Chen Y, Lin H (2015) Hyperbranched Polymer Functional TiO2 Nanoparticles: Synthesis and Its Application for the anti-UV Finishing of Silk Fabric. Fibers Polym 16(3):503-509. 
https://doi.org/10.1007/s12221-015-0503-1

42. Zhang Y, Wang J, Zhang L (2010) Creation of highly stable selenium nanoparticles capped with hyperbranched polysaccharide in water. Langmuir 26(22):17617-17623.

https://doi.org/10.1021/la1033959

43. Zhang Y, Zhang C, Guo Y, Liu D, Yu Y, Zhang B (2019) Selenium vacancy-rich $\mathrm{CoSe}_{2}$ ultrathin nanomeshes with abundant active sites for electrocatalytic oxygen evolution. Journal of Materials Chemistry A 7(6):2536-2540. https://doi.org/10.1039/C8TA11407B

44. Zheng J, Song F, Wang XL, Wang YZ (2014) In-situ synthesis, characterization and antimicrobial activity of viscose fiber loaded with silver nanoparticles. Cellulose 21(4):3097-3105. https://doi.org/10.1007/s10570-014-0324-1

45. Zhu J, Liu S, Palchik O, Koltypin Y, Gedanken A (2000) Shape-Controlled Synthesis of Silver Nanoparticles by Pulse Sonoelectrochemical Methods. Langmuir 16(16):6396-6399. https://doi.org/10.1021/la991507u

\section{Scheme}

Scheme 1,2 and 3 is available in supplementary section.

\section{Figures}
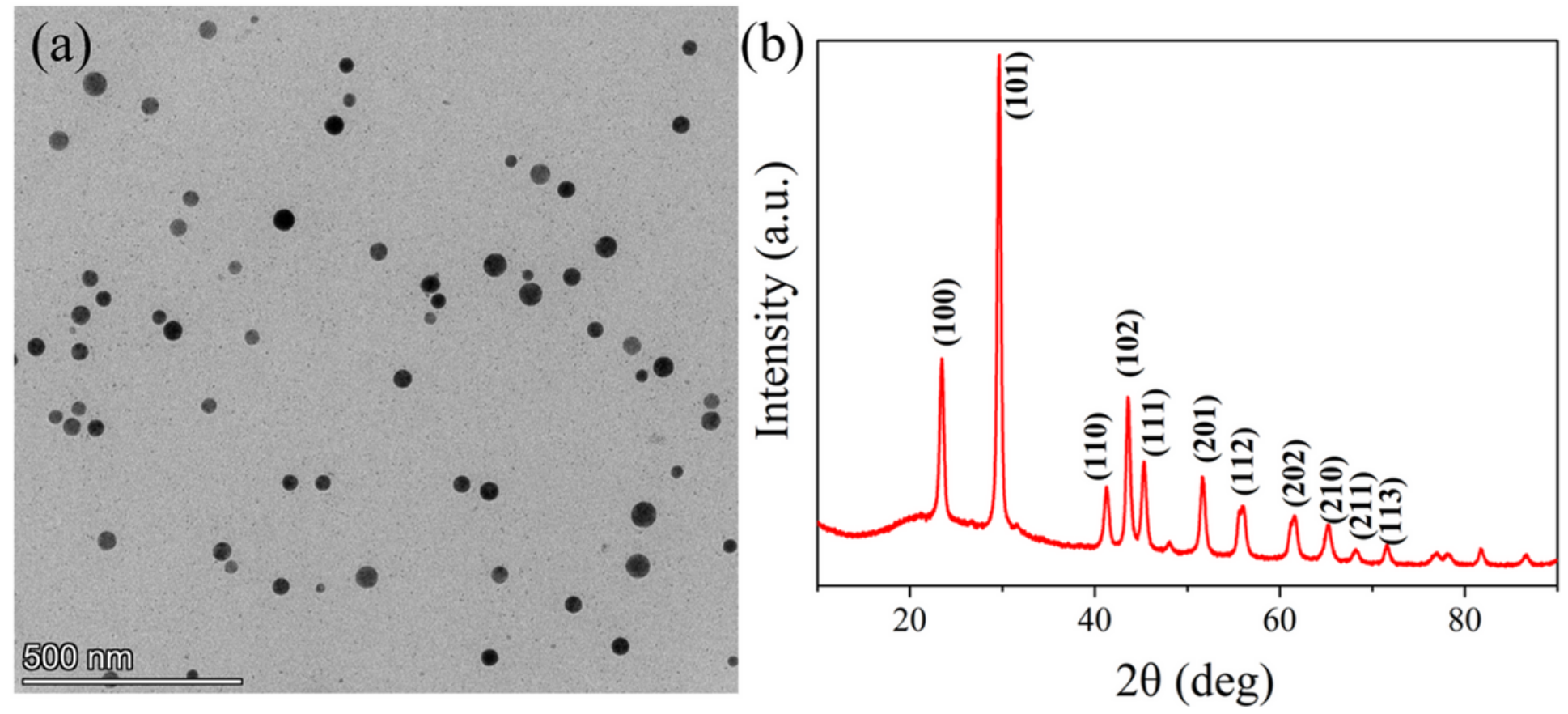

\section{Figure 1}

(a) TEM images (b) XRD of Se NPs. 


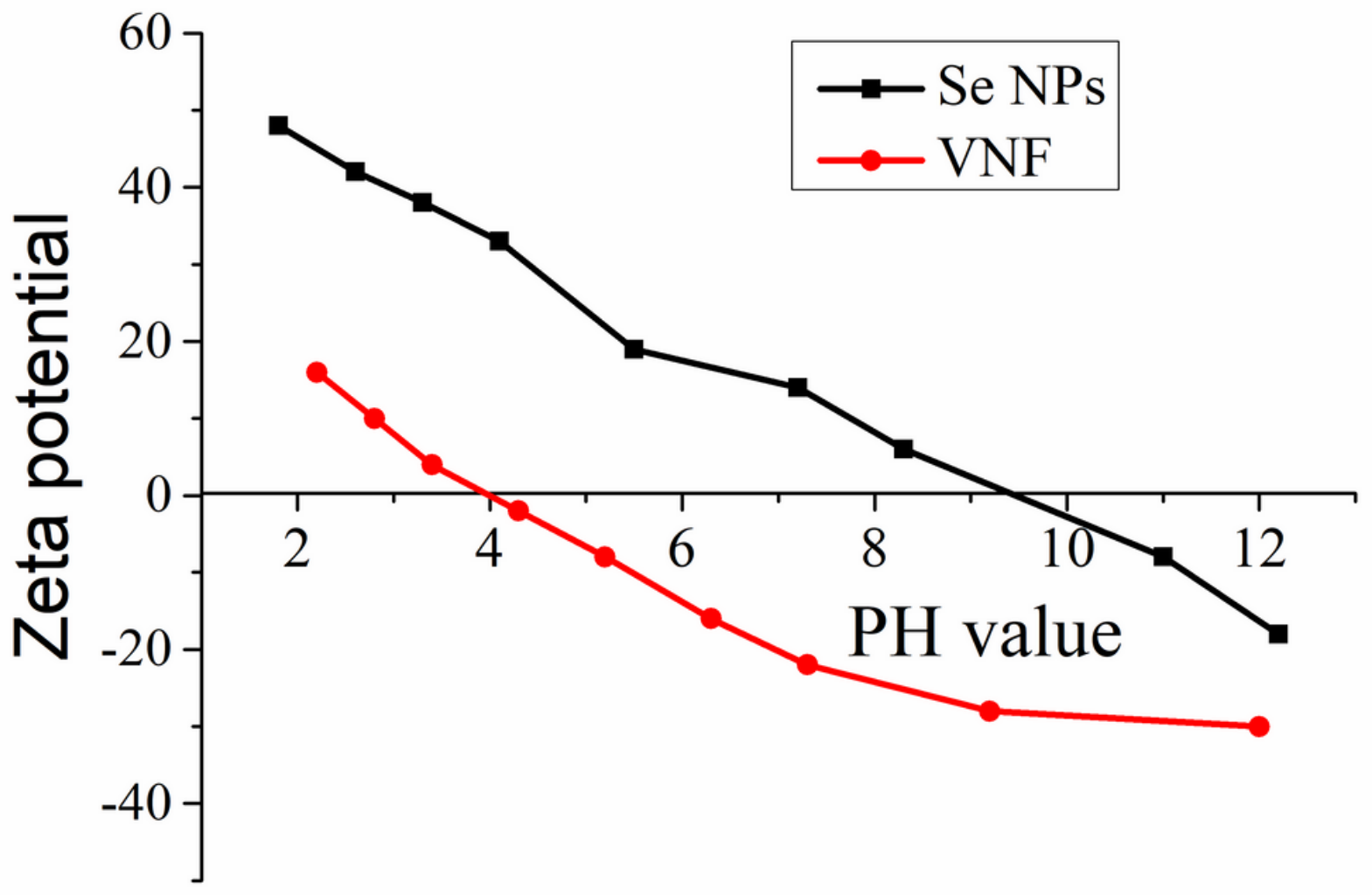

Figure 2

Zeta potential of HBP/Se NPs and VNF with different $\mathrm{pH}$ value 

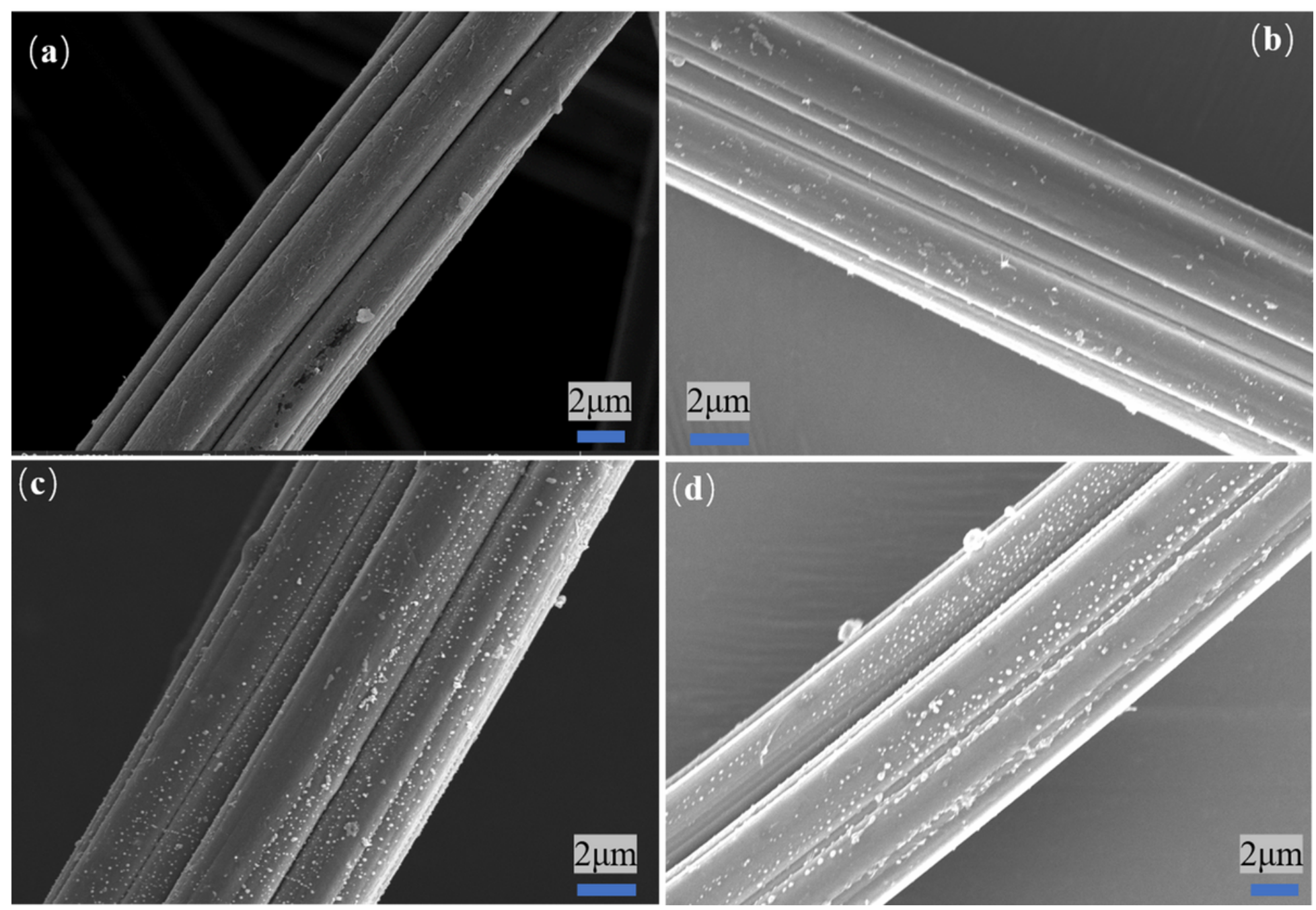

\section{Figure 3}

SEM images of (a) VNF fiber, Se NPs-coated VNF with $0.5 \mathrm{mM}, 0.1 \mathrm{mM}$, and $0.15 \mathrm{mM}$ Se NPs solution 


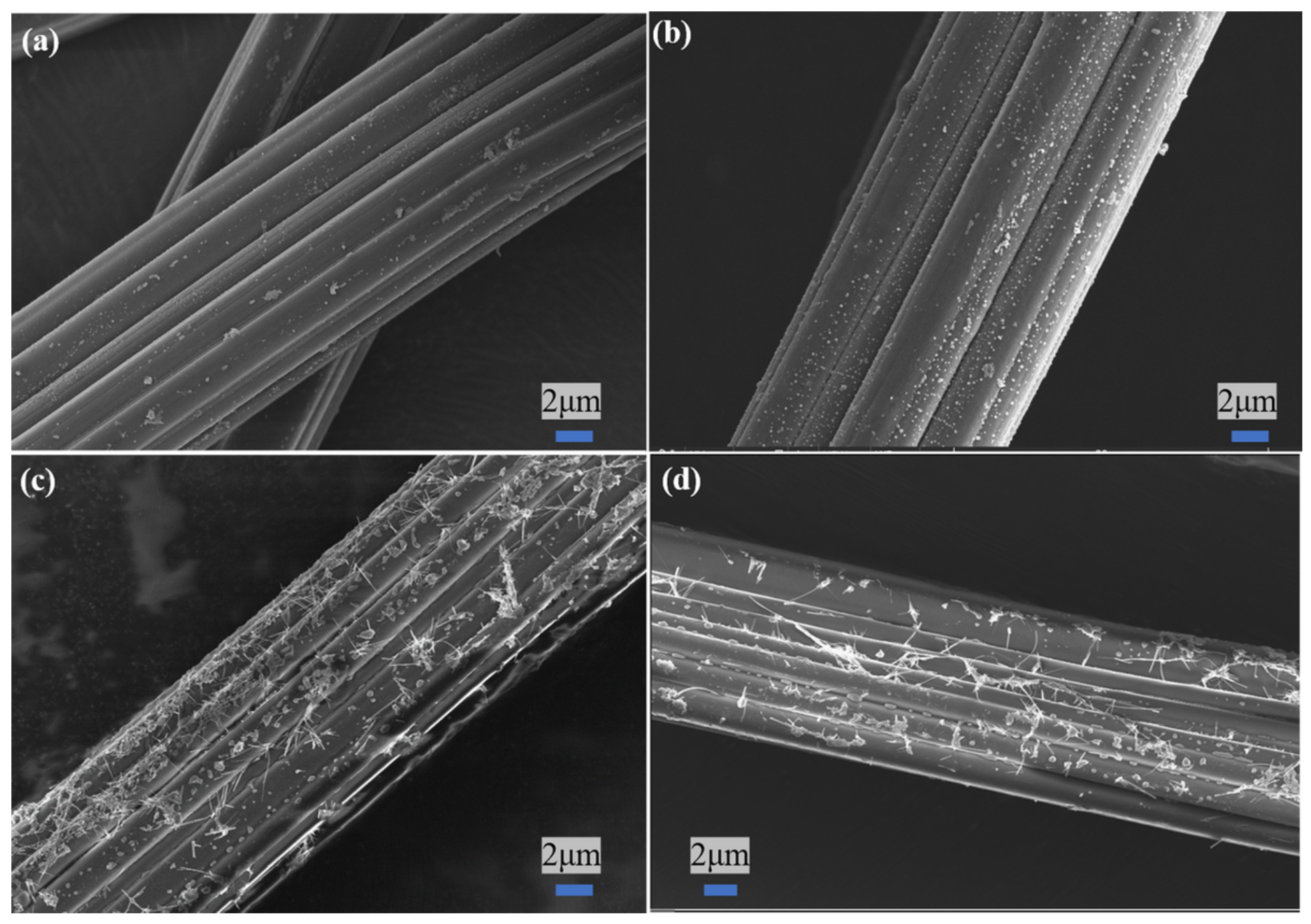

Figure 4

SEM images of (a) Se NPs-coated VNF treated with $30^{\circ} \mathrm{C}$, (b) $50{ }^{\circ} \mathrm{C}$, (c) $70{ }^{\circ} \mathrm{C}$, and (d) $90{ }^{\circ} \mathrm{C}$ 


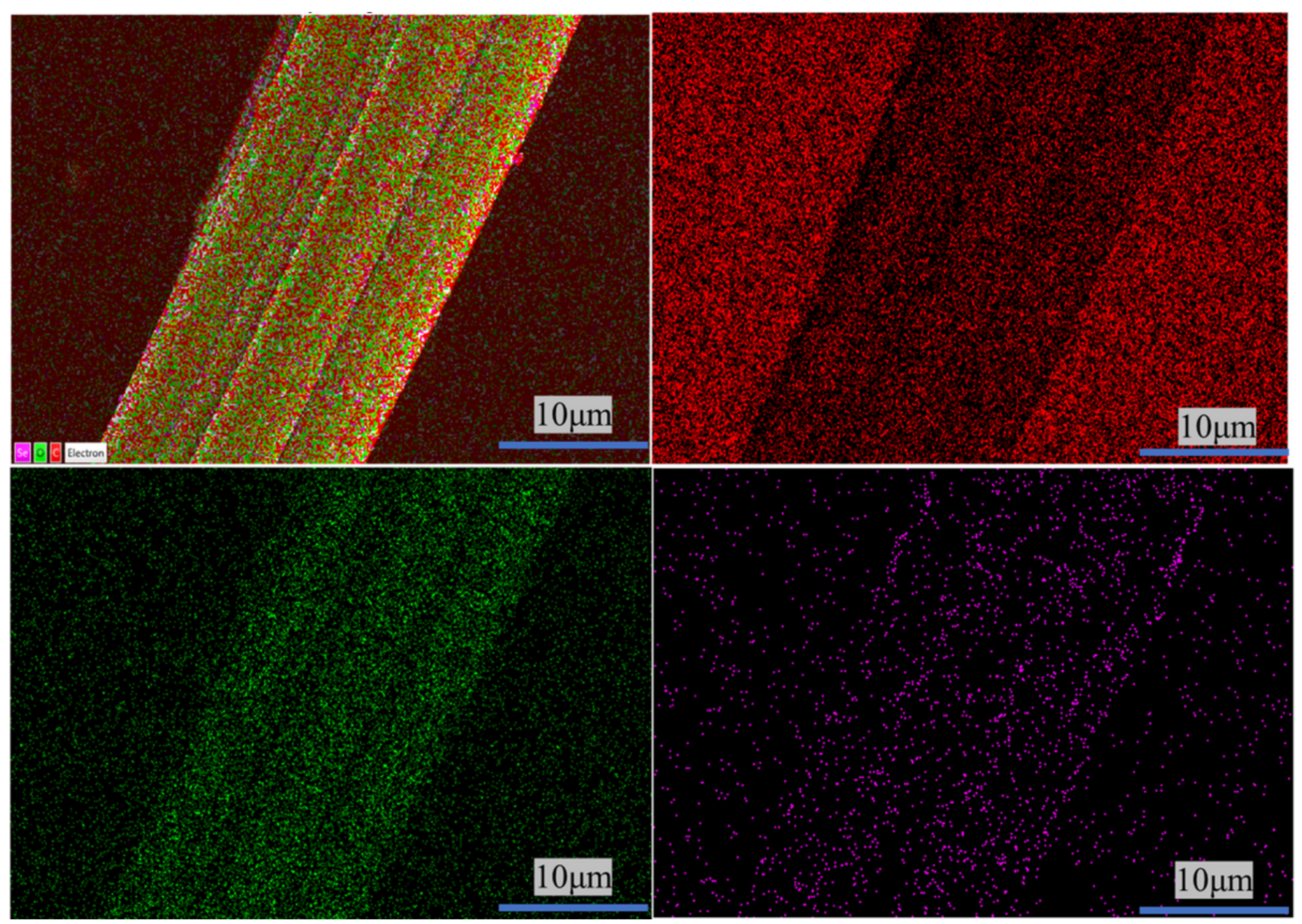

Figure 5

EDS images of HBP/Se NPs grafted VNF: (a) VNF, (b) C (c) O, and (d) Se 


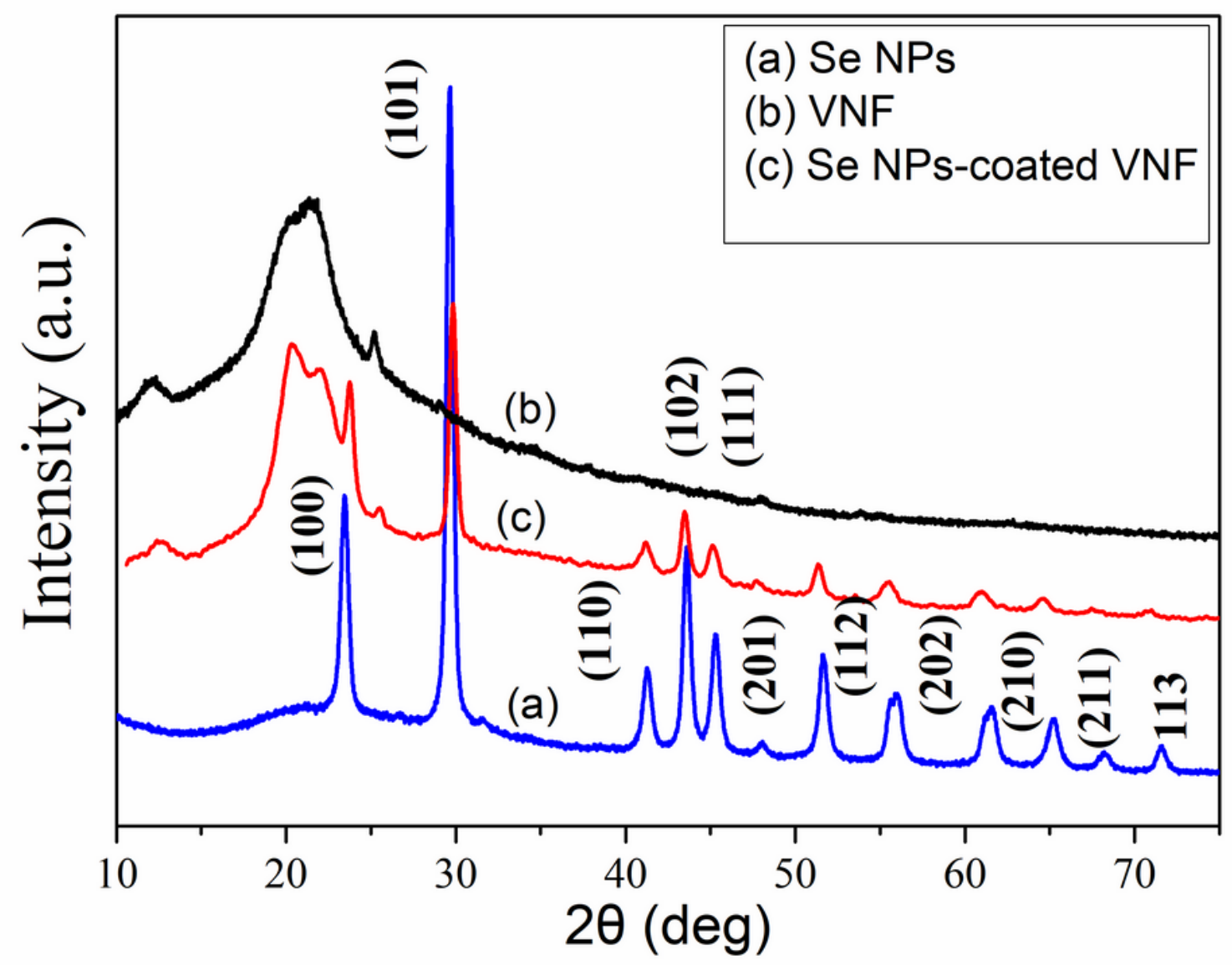

Figure 6

XRD patterns of (a) Se NPs, (b) VNF, and (c) Se NPs-coated VNF.
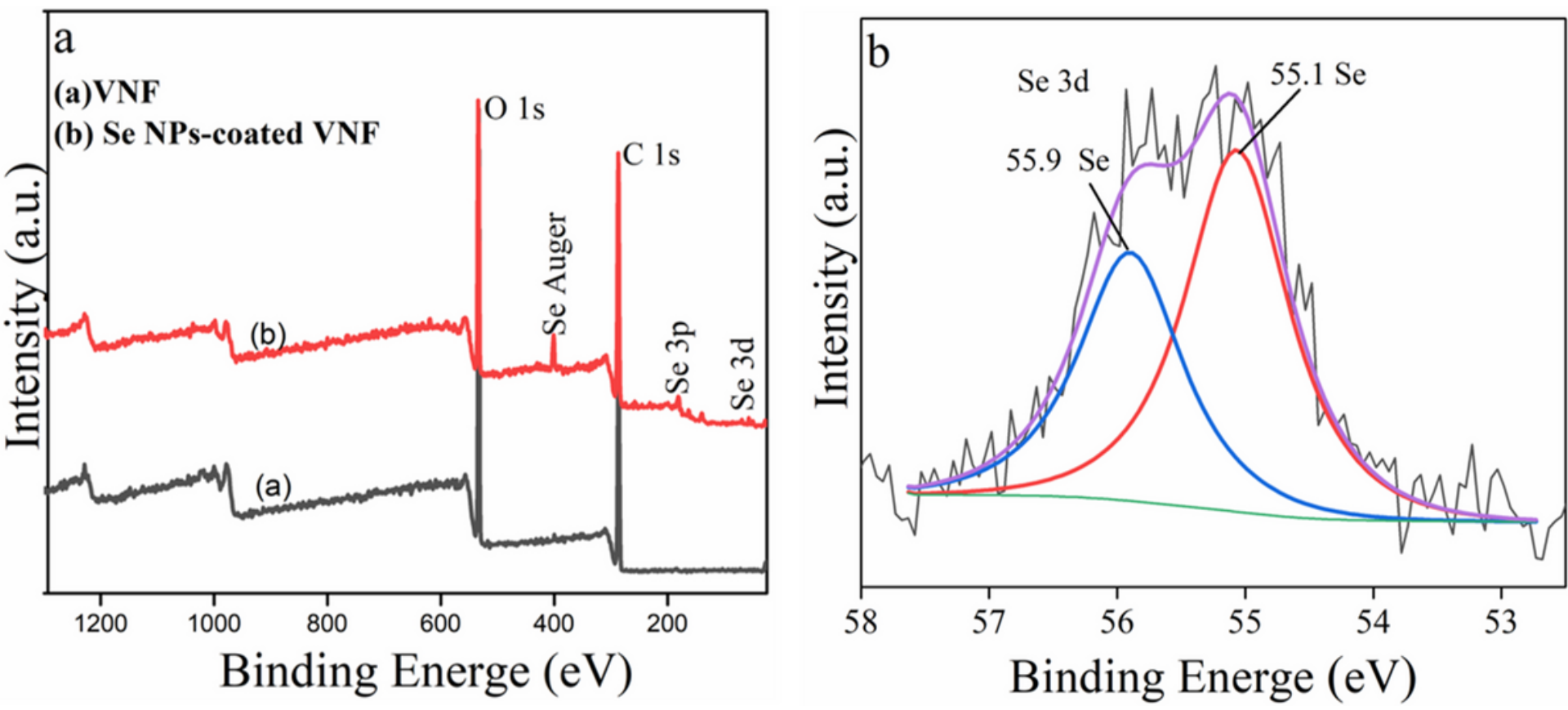
Figure 7

(a) High-resolution XPS spectra (b) Se 3d for Se NPs-coated VNF
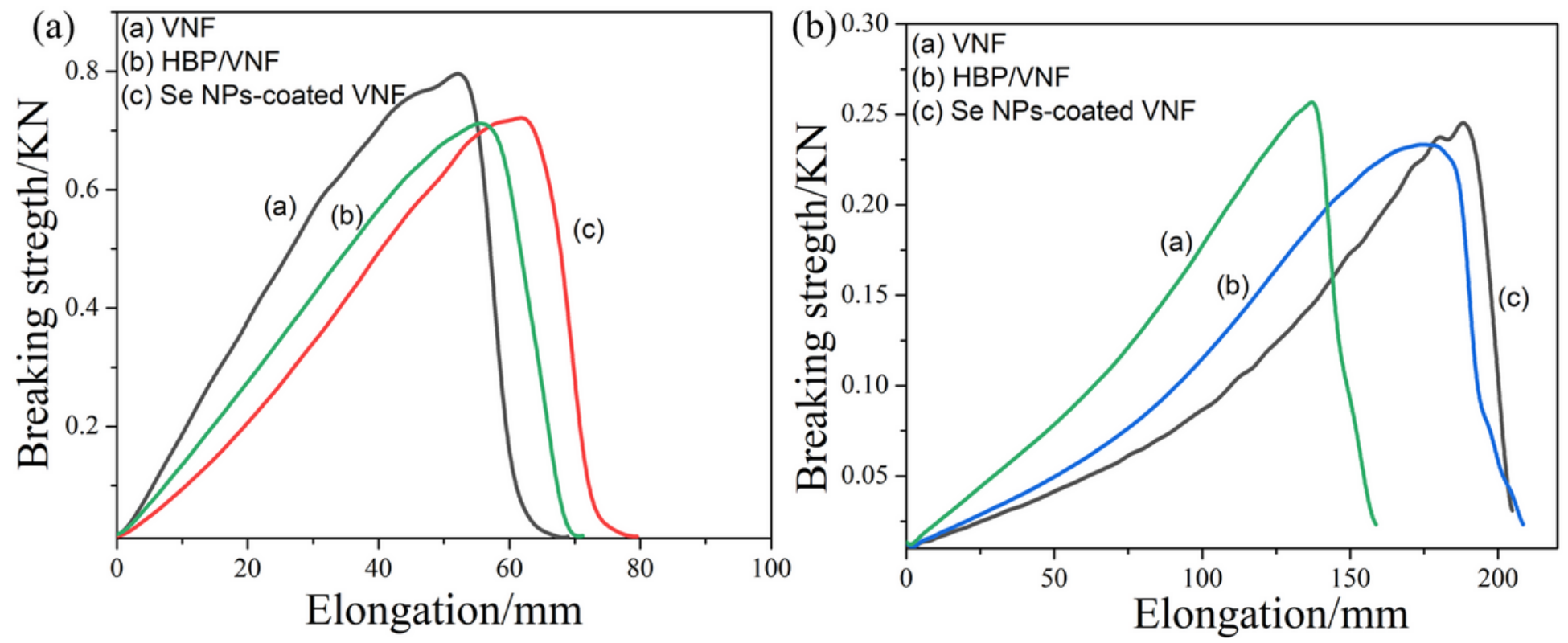

Figure 8

(a) Longitudinal breaking strength and (b) cross-breaking strength of the NWF

\section{Supplementary Files}

This is a list of supplementary files associated with this preprint. Click to download.

- Graphicabstract.tif

- suppotinginformation.docx

- OnlineSch.1.png

- OnlineSch.2.png

- OnlineSch.3.png 